\title{
POSITIVE SEMICHARACTERS OF SOME COMMUTATIVE SEMIGROUPS
}

\author{
C. W. $\operatorname{AUSTIN}^{1}$
}

1. Introduction. In their paper [3], E. Hewitt and H. S. Zuckerman considered a commutative semigroup $G$ which satisfies certain axioms, to be stated below, and which they called a "single $H_{x}$." (See, for example, Theorems 8.9 and 8.10 of [3].) As a consequence of its defining axioms, a semigroup of this type has the following properties: (1) if $x$ and $y$ are distinct elements of $G$, then $G$ has a semicharacter $\chi$ such that $\chi(x) \neq \chi(y)$; (2) the semigroup $G$ has no proper prime ideals; (3) as a result of (2), if $\chi$ is a semicharacter of $G$, then either $|\chi(x)|=1$ for all $x \in G$ or $0<|\chi(x)|<1$ for all $x \in G$.

In the present paper we consider positive real-valued semicharacters of an $H$-semigroup $G$ (that is, a single $H_{x}$; the motivation for the latter terminology is not apparent here, and the shorter term has been substituted). In some especially well-behaved cases, for example the additive semigroup of positive integers, every such semicharacter is a non-negative real power of some fixed positive semicharacter, and the set $G^{*}$ of all positive semicharacters of $G$ is isomorphic in a natural way to $R_{0}^{+}$, the additive semigroup of non-negative real numbers. We obtain a third algebraic axiom for an $H$-semigroup $G$ which is necessary and sufficient for $G^{*}$ to separate points of $G$ and to be isomorphic to $R_{0}^{+}$. We then observe that every $H$-semigroup which satisfies this axiom is actually (within isomorphism) a subsemigroup of $R^{+}$, the semigroup of positive real numbers.

Out of all this we obtain two things: a set of algebraic axioms for an abstract semigroup $G$ which suffice to imbed $G$ into $R^{+}$, and a simple algebraic characterization of all subsemigroups of $R^{+}$whose positive semicharacters are all of the form $\chi(x)=e^{-a x}$ for some $a \geqq 0$. The first result is only a curiosity; the second may be more useful.

2. $H$-semigroups.

2.1. Definition. An $H$-semigroup is a commutative semigroup $G$ which satisfies the following conditions:

(1) if $x, y \in G$ and $x^{2}=x y=y^{2}$, then $x=y$; 1963.

Received by the editors December 3, 1962 and, in revised form, February 20,

1 The results of this paper appeared in part in the author's doctoral dissertation, University of Washington, 1962, the research for which was supported by National Science Foundation Grant NSF-G10738, and received invaluable encouragement from Professors Edwin Hewitt and Herbert S. Zuckerman. 
(2) if $x, y \in G$, there exist $u, v \in G$ and a positive integer $n$ such that $x^{n}=u y$ and $y^{n}=v x$.

2.2. Definition. Let $S$ be any semigroup. A semicharacter of $S$ is a bounded, multiplicative, complex-valued function $\chi$ on $S$ which is not identically zero.

The set of all semicharacters of $S$ will be denoted by $\hat{S}$, and the set of all non-negative real-valued semicharacters of $S$ will be denoted by $S^{*}$. Clearly, $S^{*}=\{|\chi|: \chi \in \hat{S}\}$. The condition (1) of 2.1 is necessary and sufficient for $\hat{G}$ to separate points of $G[3,3.5$ and 5.8]. If $G$ satisfies (1), then the condition (2) is necessary and sufficient for $G$ to have no proper prime ideals. (A prime ideal of a semigroup is an ideal whose complement is a subsemigroup. This implies that the semigroup is not itself a prime ideal; however, the empty set is considered a prime ideal. The prime ideals of $S$ are precisely the sets $\{x \in S: \chi(x)=0\}$ for some $\chi \in \hat{S}$.) Thus (2) holds if and only if $\chi(x) \neq 0$ for all $x \in G$ and $\chi \in \hat{G}$. Since the set $\{x \in G:|\chi(x)|<1\}$ is also a prime ideal whenever $\chi \in \hat{G}$, (2) also implies that either $|\chi| \equiv 1$ or else $0<|\chi(x)|<1$ for all $x \in G$.

2.3. Definition. A proper $H$-semigroup is one which is not a group. According to $[3,4.16]$, an $H$-semigroup $G$ is proper if and only if it contains no idempotent. In fact, $G$ may contain no element of finite order (i.e., an element $x$ such that $x^{k}=x^{k+j}$ for some positive integers $k$ and $j$ ). For, if $x$ is of finite order, then some power of $x$ is idempotent $[2,2.6 .2]$.

The following theorem is Theorem 8.10 of [3], quoted here without proof.

2.4. Theorem. Let $G$ be an $H$-semigroup. Then $G$ is proper if and only if $\hat{G}$ contains an element $\chi$ with $0<|\chi(x)|<1$ for all $x \in G$.

We now observe that either $G^{*}=\{1\}$, in which case $G$ is a group, or else $0<f<1$ whenever $1 \neq f \in G^{*}$. A final important fact about $H$ semigroups is the following $[3,4.15 .1]$ :

\subsection{Theorem. The cancellation law holds in every $H$-semigroup.}

3. Linear semigroups. Throughout this section, $G$ will denote a cancellative commutative semigroup without identity (hence with no idempotents), and $N$ will denote the set of positive integers.

3.1. Definition. Let $x, y \in G$. We define $x<y$ if and only if there exist $n \in N$ and $u \in G$ such that $x^{n}=u y^{n}$.

3.2. Lemma. The relation $\prec$ is irreflexive and transitive. If $G$ is totally ordered by $\prec$, then $G$ is a positively ordered semigroup in the 
sense of [1]: that is, for all $x, y, z \in G$ we have $x z<y z$ whenever $x<y$, and also we have $x y<x$.

The proof of 3.2 is routine and is omitted.

3.3. Lemma. Let $x \in G$. If $x^{m}=u x^{n}$ for some $u \in G$ and $m, n \in N$, then $m>n$.

Proof. If $x^{m}=u x^{m}$, then $u^{2}=u$. If $x^{m}=u x^{m+p}$ for some $p \in N$, then $u x^{p}$ is idempotent.

3.4. Definition. If $x, y \in G$ are such that none of the statements $x \prec y, x=y, y \prec x$ is true, then $x$ and $y$ are said to be noncomparable.

3.5. Lemma. Let $x, y$ be noncomparable elements of $G$. If there exist $m, n \in N$ and $u \in G$ such that $x^{m}=u y^{n}$, then $m>n$.

Proof. If $m=n$ then $x<y$; if $m+p=n$, then $x^{m}=\left(u y^{p}\right) y^{m}$ and again $x<y$.

3.6. Lemma. Let $x$ and $y$ be noncomparable elements of $G$.

(1) If $x^{m}=y^{n}$ for some $m, n \in N$, then $m=n$.

(2) If $x^{m} y^{n}=x^{p} y^{q}$ for some $m, n, p, q \in N$, then $m+n=p+q$.

(3) If $x^{m} y^{n}=x^{p} y^{q} u$ for some $m, n, p, q \in N$ and $u \in G$, then $m+n$ $>p+q$.

Proof. (1) If $x^{m}=y^{n}$, then $x^{m+1}=x y^{n}$. By 3.5 we have $m+1>n$, and similarly $n+1>m$.

(2) Let $x^{m} y^{n}=x^{p} y^{q}$. If $m=p$, then $y^{n}=y^{q}$ and so $n=q$. If $m<p$ then $y^{n}=x^{p-m} y^{q}$. By 3.3 we have $q<n$, so that $x^{p-m}=y^{n-q}$. Now $p-m$ $=n-q$ by (1).

(3) Let $x^{m} y^{n}=x^{p} y^{q} u$. If $m<p$, then $y^{n}=\left(x^{p-m} u\right) y^{q}$, so that $n>q$ by 3.3. Thus $y^{n-q}=x^{p-m} u$ and $n-q>p-m$ by 3.5. If $m=p$, then $y^{n}=y^{q} u$, and $n>q$ by 3.3. Finally, if $m>p$, then $x^{m-p} y^{n}=y^{q} u$. If $n \geqq q$ we are done; if not, then $x^{m-p}=y^{q-n} u$ and $m-p>q-n$ by 3.5.

The following result is quoted without proof.

3.7. THEOREM (K. A. Ross [5]). Let S be any commutative semigroup and let $T$ be a subsemigroup of $S$. If $\chi$ is a semicharacter of $T$, then $\chi$ can be extended to a semicharacter of $S$ if and only if $|\chi(a)| \geqq|\chi(b)|$ whenever $a, b \in T$ and $a x=b$ for some $x \in S$.

The results of 3.1 through 3.6 were originally stated for a proper $H$-semigroup, and their validity for the larger class of semigroups here considered was kindly pointed out by the referee, who also observed that the following theorem could be extracted from the original proof of 3.13 . 
3.8. THEOREM. If $x$ and $y$ are noncomparable elements of $G$ and if $0<r<1$, then there exists $f \in G^{*}$ such that $f(x)=f(y)=r$.

Proof. Let $H$ be the subsemigroup of $G$ generated by $x$ and $y$, and define $g$ on $H$ by $g\left(x^{n}\right)=r^{n}, g\left(y^{m}\right)=r^{m}$ and $g\left(x^{n} y^{m}\right)=r^{n+m}$. Then $g$ is well defined, by 3.6(1) and (2) and the fact that $G$ has no elements of finite order. Clearly $g \in H^{*}$.

If $a, b \in H$ such that $a u=b$ for some $u \in G$, it follows from 3.3, 3.5 and $3.6(3)$ that $g(a) \geqq g(b)$. Thus, by Ross' theorem, there exists a semicharacter $\chi$ of $G$ such that $\chi(u)=g(u)$ for all $u \in H$. Let $f=|\chi|$.

3.9. Theorem. Let $G$ be totally ordered by $\prec$. Then $G$ is archimedean (see [1]) if and only if $G$ is an $H$-semigroup.

Proof. Let $G$ be an $H$-semigroup. Given $x, y \in G$ we must find $n \in N$ such that $x^{n}<y$. By 2.1(2) there exist $n \in N$ and $u, v \in G$ such that $x^{n}=u y$ and $y^{n}=v x$. Thus $x^{n}<y$, and in fact $y^{n}<x$ also.

Conversely, let $G$ be archimedean. It suffices to show that $G$ has no proper prime ideal. If $J$ is a prime ideal of $G$ and $x \in J, y \in G \backslash J$, then $y^{n}=u x^{n}$ is impossible. Hence we must have $x \prec y$. But if $y \in G \backslash J$ then also $y^{n} \in G \backslash J$, so that $x \prec y^{n}$ for all $n \in N$, and $G$ is not archimedean.

3.10. Definition. A linear semigroup is (1) a proper $H$-semigroup which is totally ordered by $\prec$ or, equivalently, (2) a cancellative commutative semigroup without identity, which is totally ordered by $\prec$ and is archimedean.

3.11. Definition. We say that $G^{*}$ is one-parameter if there exists $f_{0} \in G^{*}$ such that $0<f_{0}<1$ and $G^{*}=\left\{f_{0}^{a}: a \geqq 0\right\}$.

We note that if $G^{*}$ is one-parameter, then $G$ is necessarily an $H$ semigroup because a semicharacter of $G$ vanishes nowhere.

3.12. Lemma. Let $G^{*}$ be one-parameter and separate points of $G$. If $1 \neq g \in G^{*}$ and $x, y \in G$, then $g(x)=g(y)$ implies that $x=y$.

Proof. Let $G^{*}=\left\{f_{0}^{a}: a \geqq 0\right\}$. Then $g=f_{0}^{a}$ for some $a>0$, and so $f_{0}(x)=f_{0}(y)$. Then $f(x)=f(y)$ for all $f \in G^{*}$, and $x=y$.

3.13. Theorem. Let $G$ be a cancellative commutative semigroup without identity. Then $G$ is linear if and only if $G^{*}$ is one-parameter and separates points.

Proof. If $G^{*}$ is one-parameter and separates points, then $G$ is a proper $H$-semigroup and $f(x)=f(y)$ implies that $x=y$ whenever $1 \neq f \in G^{*}$. If $G$ had noncomparable elements $x, y$, then by 3.8 there would exist $f \in G^{*}$ with $f(x)=f(y)=r \neq 1$. 
Conversely, suppose that $G$ is linear. Then there exists $f \in G^{*}$ such that $0<f<1$. Clearly $x<y$ implies that $f(x)<f(y)$, so that $G^{*}$ separates points.

If $G^{*}$ is not one-parameter, there exist $g \neq 1$ and $h \neq 1$ in $G^{*}$ and $x, y \in G$ with $g(x)=h(x)^{a}$ and $g(y)=h(y)^{b}$ for some distinct $a$ and $b$. We may suppose that $a<b$. Let $c>0$ be such that $h(x)=h(y)^{c}$, and let $r, s \in N$ be such that $1 / c<r / s<b / a c$. Let $u=x^{2 r} y^{*}$ and $v=x^{r} y^{2 e}$. Since $s<r c$ and $r a c<s b$, it follows that:

(1) $h(u)=h(y)^{2 r c+s}<h(y)^{r c+2 s}=h(v)$, and

(2) $g(u)=h(y)^{2 r a c+s b}>h(y)^{r a c+2 s b}=g(v)$.

But $u$ and $v$ must be comparable, which implies that $g(u) \leqq g(v)$ and $h(u) \leqq h(v)$ or vice versa.

4. Subsemigroups of $R^{+}$.

4.1. Theorem. Every linear semigroup is isomorphic to a subsemigroup of $R^{+}$.

Proof. Let $G$ be a linear semigroup. Then there exists $f \in G^{*}$ such that $0<f<1$. Since $x<y$ implies that $f(x)<f(y)$, it follows that the mapping $x \rightarrow-\log f(x)$ is an isomorphism of $G$ into $R^{+}$.

If $G \subset R^{+}$and $x, y \in G$ then $x<y$ if and only if $n x=u+n y$ for some $u \in G$ and $n \in N$; in other words, $x>y$ and $n(x-y) \in G$ for some $n \in N$. This observation leads to the following result.

4.2. Theorem. Let $G$ be a subsemigroup of $R^{+}$. Then the following are equivalent.

(1) If $f \in G^{*}$, then there exists $a \geqq 0$ such that $f(x)=e^{-a x}$ for all $x \in G$.

(2) If $x, y \in G$ and $y<x$, then $n(x-y) \in G$ for some $n \in N$.

Proof. If (1) is true, then $G^{*}$ is one-parameter and separates points, so that $G$ is linear. If $x, y \in G$ with $y<x$, then we must have $x<y$, so that (2) holds.

Conversely, if (2) holds then $G$ is totally ordered by $\prec$. If $x, y \in G$ then $n x>y$ for some $n \in N$, and hence $n x<y$. Thus $G$ is archimedean (with respect to $\prec$ ) and is therefore linear. Hence $G^{*}$ is one-parameter, and (1) follows.

4.3. Examples. Among the subsemigroups of $R^{+}$which satisfy 4.2(2) are the following: $R^{+} ; Q^{+}$, the additive semigroup of positive rationals; $N$; for any $a>0$ the semigroups $\left.R^{+} \cap\right] a, \infty\left[, Q^{+} \cap\right] a, \infty[$ and $N \cap] a, \infty$ [; any semigroup which contains ] $a, \infty$ [ for some $a>0$, and in particular, any subsemigroup of $R^{+}$which has positive Lebesgue measure (see [4, Theorem 7.3.2]). We note in passing that a positive semicharacter of $R^{+}$is necessarily continuous, being of the form $x \rightarrow e^{-a x}$. 
The very short proof of 4.1 depends on the nontrivial result of Hewitt and Zuckerman [3, Theorem 8.10] that a proper $H$-semigroup always has a semicharacter $f$ such that $0<f<1$. The use of this fact is avoided in the following argument, suggested by the referee: let $G$ be a linear semigroup, $H$ its group of quotients, and $G^{\prime}$ the set of $x$ in $H$ such that $x^{n} \in G$ for some $n \in N$. In addition to being positively ordered and archimedean, $G^{\prime}$ is also naturally ordered and hence, by a theorem of Hölder and Huntington (see [1]), $G^{\prime}$ is isomorphic to a subsemigroup of $R^{+}$; so is $G$.

\section{REFERENCES}

1. A. H. Clifford, Totally ordered commutative semigroups, Bull. Amer. Math. Soc. 64 (1958), 305-316.

2. E. Hewitt and H. S. Zuckerman, Finite dimensional convolution algebras, Acta Math. 93 (1955), 67-119.

3. - The $h_{1}$-algebra of a commutative semigroup, Trans. Amer. Math. Soc. 83 (1956), 70-97.

4. E. Hille and R. S. Phillips, Functional analysis and semigroups, Amer. Math. Soc. Colloq. Publ. Vol. 31, Amer. Math. Soc., Providence, R. I., 1957.

5. K. A. Ross, $A$ note on extending semicharacters of semigroups, Proc. Amer. Math. Soc. 10 (1959), 597-583.

UnIVERSITY OF CoLORADO

\section{REPRESENTATIVE SETS AND DIRECT SUMS}

\section{ROBERT RYAN ${ }^{1}$}

1. Let $(X, \Lambda, \mu)$ be a complete measure space and denote by $N$ the collection of all locally $\mu$-null subsets of $X$, i.e., $E \in N$ if and only if $\mu(E \cap F)=0$ for all $\mu$-summable sets $F$. For $E$ and $F$ in $\Lambda$ we write $E \equiv F$ if and only if $E \Delta F \in N$ where $E \Delta F=(E-F) \cup(F-E)$. The relation $E \equiv F$ is clearly an equivalence relation.

$J$. von Neumann showed that if $\mu$ is $\sigma$-finite there exists a mapping $\rho$ of $\Lambda$ into $\Lambda$ with the following properties:

(1) $\rho(E) \equiv E$;

(2) $E \equiv F$ implies $\rho(E)=\rho(F)$;

(3) $\rho(\varnothing)=\varnothing, \rho(X)=X$ ( $\varnothing=$ the empty set);

(4) $\rho(E \cap F)=\rho(E) \cap \rho(F)$;

(5) $\rho(E \cup F)=\rho(E) \cup \rho(F)$.

Received by the editors January 10, 1963 and, in revised form, February 18, 1963.

1 Sponsored by the Mathematics Research Center, U. S. Army, Madison, Wis consin, under Contract No. DA-11-022-ORD-2059. 